\title{
Avaliação da atenção prestada aos familiares em um centro de atenção psicossocial
}

Evaluation of family care delivered at a psychosocial care center

Evaluación de la atención prestada a los familiares en un centro de atención psicosocial

\section{Maria de Lourdes Custódio Duarte', Luciane Prado Kantorski'}

'Universidade Federal de Pelotas. Escola de Enfermagem. Pelotas, RS

Submissão: 22/09/2009

Aprovação: 12/09/2010

\section{RESUMO}

Este estudo objetivou avaliar Qualitativamente a atenção oferecida aos familiares por um Centro de Atenção Psicossocial (CAPS) do tipo I, situado na Região Sul do Brasil. O Círculo Hermenêutico Dialético foi utilizado como técnica de coleta de dados, método preconizado pela Avaliação de Quarta Geração proposta por Guba e Lincoln. Temas como acolhimento, reuniões de familiares, visitas domiciliares e associação dos familiares surgiram nas entrevistas com os familiares do CAPS. Entrevistas e observações foram as técnicas utilizadas para a coleta de dados. A forma como vem sendo consolidada a atenção às famílias nos serviços representa um desafio para os profissionais de saúde, por implicar reestruturação na formação destes e um processo de reflexão de todos os atores sociais envolvidos neste processo de mudança.

Descritores: Avaliação de serviços de saúde; Saúde mental; Família.

\section{ABSTRACT}

This study aimed to evaluate Qualitatively the care offered to families for a Center of Psychosocial Atention of type I, located in Southern Region of Brazil. The Dialectical hermeneutic circle was used as a technique of data collection, a method recommended by the Fourth Generation Evaluation proposed by Guba and Lincoln. Topics such as reception, family meetings, home visits and involvement of family emerged in interviews with family members of this health service. Interviews and observations were the technieues used to collect data. The way has been consolidated to care for families in services represents a challenge for health professionals, since it entails the restructuring and formation of a reflection of all the actors involved in this process of change.

Key words: Health services evaluation; Mental health; Family.

\section{RESUMEN}

Este estudio tuvo como objetivo evaluar cualitativamente la atención ofrecida a las familias para un tipo de Centro de Atención Psicosocial del tipo I, situada en el la Region Sur de Brasil. El círculo hermenéutico dialéctico se utilizó como técnica de recolección de datos, un método recomendado por la cuarta generación de evaluación propuesto por Guba y Lincoln. Temas tales como la recepción, reuniones familiares, visitas a domicilio y la participación de la familia surgieron en las entrevistas con los familiares de estos servicios de salud. Las entrevistas y observaciones fueron las técnicas utilizadas para recoger datos. El camino se ha consolidado a la atención de las familias en los servicios representa un reto para los profesionales de la salud, ya Que implica la reestructuración y la formación de una reflexión de todos los actores involucrados en este proceso de cambio.

Descritores: Evaluación de servicios de salud; Salud mental; Familia. 


\section{INTRODUÇÃO}

A partir da década de 1960, os serviços passaram a ser pensados também como produtos e, portanto, passíveis de uma padronização na Qualidade, atualmente um atributo importante na valorização do mercado. A Qualidade dos serviços passou a ser encarada como parte dos direitos sociais da população não só do setor privado, mas, também, do setor público ${ }^{(1)}$. Nesse contexto, os serviços de saúde tornaram-se alvo de avaliação tanto pelos órgãos do governo Quanto pelos próprios consumidores e clientes.

A avaliação é um processo importante para o planejamento estratégico à medida Que permite a formulação de juízos com o objetivo de chegar a conclusões fundamentadas Que subsidiem ações futuras. Dessa forma, a avaliação somente se justifica Quando permite uma retroalimentação dos processos em curso, a fim de corrigir, sanar ou evitar eventuais erros, estabelecendo estratégias para melhorar a Qualidade da assistência prestada ${ }^{(2)}$.

No Brasil, a importância da avaliação das políticas públicas é reconhecida em documentos oficiais e científicos, mas esse reconhecimento ainda não se traduz em processos de avaliação sistemáticos e consistentes Que subsidiem a gestão pública ${ }^{(3)}$. Em verdade, notase Que o Brasil ainda não tem o costume de avaliar suas políticas, programas e, tampouco, seus serviços de saúde.

No campo da saúde mental, nos últimos anos também houve um visível interesse pelo tema da avaliação em saúde, intensificando pelo notável aparecimento de novos serviços, decorrentes de um amplo processo de transformações denominado Reforma PsiQuiátrica.

É nesse cenário de transformações na Saúde Mental, encabeçados pela Reforma PsiQuiátrica, Que os novos serviços passaram a ser alvo de avaliações. A maior parte dos estudos envolvendo avaliação no campo da saúde mental está relacionada a parâmetros epidemiológicos clássicos (modelos estatísticos) e a auditorias externas, com o objetivo de verificação do bom funcionamento dos serviços e Que na prática não tem como finalidade a transformação para uma melhoria do serviço ${ }^{(4)}$. O Que se pretende com este processo avaliativo é conseguir captar Questões Que com o método Quantitativo seria inviável. Questões envolvendo subjetividade e complexidade do são captadas através de métodos Qualitativos, indo muito além de dados epidemiológicos Quantificáveis.

Uma das inúmeras mudanças preconizadas pela Reforma Psiquiátrica foi a que o sujeito em sofrimento psíquico deve ser assistido em um sistema comunitário de saúde mental denominado de Centros de Atenção Psicossocial (CAPS), Que visam a desinstitucionalização, a reabilitação psicossocial e a reinserção social deste indivíduo.

Os CAPS foram formalmente definidos pela Portaria $\mathrm{n}^{\circ} 224 \mathrm{de}$ 29 de janeiro de 1992, do Ministério da Saúde como unidades locais/regionalizadas, Que contam com uma população adscrita definida pelo nível local e Que oferecem atendimento de cuidados intermediários entre o regime ambulatorial e a internação hospitalar por uma equipe multiprofissional ${ }^{(5)}$. Na realidade, a equipe multiprofissional dos CAPS deve direcionar suas intervenções no sentido de buscar reabilitar o sujeito reintegrando-o na sua família e comunidade.

Esses serviços deverão também estar capacitados para realizar prioritariamente o atendimento de usuários com transtornos mentais graves e persistentes em sua área territorial, em regime de tratamento intensivo, semi-intensivo e não intensivo. Os CAPS possuem três níveis de funcionamento de acordo com a portaria 336 , de 19 de fevereiro de $2002^{(5)}$ : CAPS 1, CAPS 2 e CAPS 3, definidos por ordem crescente de porte, complexibilidade e abrangência populacional. Na realidade, as três modalidades de atendimento cumprem a mesma função no atendimento público em saúde mental, devendo-se constituir em um serviço ambulatorial de atenção diária Que evite a internação em hospital psiQuiátrico.

O processo de desinstitucionalização, preconizado pela Reforma Psiquiátrica, fez nascer a necessidade de reflexão sobre essa nova política de assistência. A pesquisa avaliativa nesse cenário deve possibilitar a inclusão de diferentes opiniões e valores dos envolvidos com os novos serviços. Deve, também, viabilizar e ampliar a utilização dos resultados da avaliação e, principalmente, "empoderar" os atores sociais envolvidos (trabalhadores e usuários), possibilitando Que se apropriem dos conhecimentos ligados à realização de um processo avaliativo ${ }^{(4)}$.

Uma das mudanças proporcionadas pela reestruturação da assistência psieuiátrica foi a de estimular a família a participar do cuidado junto aos serviços de saúde, possibilitando ao usuário permanecer o maior tempo possível junto a sua unidade de cuidado. Com essas transformações, os serviços de saúde mental passaram a formar parcerias de cuidado, fazendo com Que as famílias, assumissem um papel importante e ativo no tratamento, estabelecendo assim, uma rede de relações entre família, usuário, serviço de saúde e sociedade.

Os familiares, por sua vez, têm dificuldades em Questionar a atenção oferecida pelos profissionais pelo receio de haver uma interferência negativa no cuidado dispensado ao seu familiar enfermo. Os profissionais de saúde mental precisam estar atentos às suas atitudes e à forma de tratar os familiares, devendo melhorar a cooperação e o suporte oferecido ao sistema familiar, e, principalmente, criar oportunidades de encontros entre os familiares de sujeitos com transtornos mentais ${ }^{(6)}$.

A família é, na maioria das vezes, a unidade de saúde para seus membros, pois é ela Que, de forma explícita, cuida da saúde de seus integrantes. Isso faz com que essa unidade de cuidado tornese um referencial para os serviços de saúde, na busca de informações para a identificação do problema ${ }^{(7)}$.

Com a desinstitucionalização do louco, as práticas assistenciais foram, aos poucos, visualizando a família do portador de transtorno mental como um grande aliado, passando a dividir a responsabilidade pelo cuidado e pela inserção social. Já, no modelo manicomial, a família era excluída do tratamento e chegou a ser considerada por Pinel como a causadora da doença, porQue a alienação poderia ter a influência de uma educação corrompida e do desregramento no modo de viver ${ }^{(8)}$.

O novo papel da família foi reafirmado no Relatório Final da $3^{\text {a }}$ Conferência Nacional de Saúde Mental( ${ }^{(9)}$, afirmando Que os serviços substitutivos, como os CAPS, deveriam incluir ações dirigidas aos familiares e ao portador do transtorno mental, visando a inserção social e respeitando as particularidades individuais. Assim, a família é chamada a uma conscientização de seus direitos, viabilizando o exercício da sua cidadania.

Observa-se Que a atenção em saúde está atrelada ao cuidado humanizado integral, ao acolhimento e à criação de vínculo. Se 
todas essas premissas forem contempladas pelos trabalhadores em saúde, melhorará a Qualidade dos serviços de saúde, satisfazendo os usuários e familiares. A atenção à saúde e o sistema de saúde objetivam conferir aos indivíduos, família e comunidade cuidado humanizado nas ações e serviços de saúde, ampliando máxima Qualidade de assistência, acolhimento das pessoas e a resolutividade de cada caso ${ }^{(10)}$.

A atenção humanizada, preconizada pelo SUS significa um avanço no Que se refere às políticas públicas, entretanto, há necessidade também de uma atenção integral do cuidado prestado ao usuário, bem como a Qualidade técnica prestada da assistência, melhorando o acolhimento das pessoas e a capacidade resolutiva de cada problema $^{(11)}$. É nessa perspectiva Que os CAPS devem atuar.

Avaliar a atenção oferecida aos familiares num CAPS, objeto deste estudo, prevê a participação dos familiares envolvidos, mostrando-lhes sua importância no direcionamento de melhorias da Qualidade dos serviços prestados, valorizando as diferentes opiniões e valores daQueles Que utilizam o serviço.

Com a inclusão da família no setting do tratamento, assumindo diversos papéis, o de agente terapêutico e de facilitador, faz com Que essa unidade de cuidado assuma responsabilidades o que the dá a condição e a Qualifica ser agente avaliadora dos serviços prestados pelos CAPS. É nessa perspectiva Que esse estudo se centra, visualizando a unidade familiar como agente avaliador e Qualificado para assumir tal posição, tornando-a, conseQuentemente, um grupo de interesse com mais poder político em relação ao serviço.

Ao avaliar a atenção oferecida aos familiares por um serviço de saúde, o CAPS, pode ser uma estratégia para tornar essas instituições mais eficazes no Que se propõem a fazer, como estimular a participação da família no tratamento do seu familiar enfermo nos serviços de Saúde Mental.

Portanto, este estudo torna-se relevante por realizar uma avaliação Qualitativa da atenção à família em um serviço substitutivo, tendo por objetivo avaliar Qualitativamente a atenção oferecida aos familiares por um CAPS da região sul do Brasil, identificando, na proposta desse serviço, espaços e atividades de suporte aos familiares de portadores de transtornos psíquicos.

\section{MÉTODOS}

Este estudo foi realizado em um CAPS tipo I, localizado no extremo sul do Estado do Rio Grande do Sul. O local do estudo foi escolhido pelo seu pioneirismo, pois foi um dos primeiros serviços substitutivos aos hospitais psieuiátricos na região sul do país e também por ser considerado um serviço modelo de assistência para outros serviços da região. Vale ressaltar que o CAPS desenvolve uma assistência não-manicomial preconizada pela Reforma PsiQuiátrica, desde 1988.

Tratou-se de uma avaliação construtivista, responsiva, com abordagem hermenêutica-dialética, denominada Avaliação de Quarta Geração ${ }^{(10)}$. Caracteriza-se, também, por ser descritivo e analítico, com abordagem Qualitativa dos dados. Também será descritivo poreue observará, registrará e correlacionará fatos sem manipulá-los.

A Avaliação de Quarta Geração é uma forma de avaliação em Que as reivindicações, preocupações e Questões do grupo de interesse (Stakeholders) servem de base para determinar a informação necessária, a Qual é implementada através dos pressupostos metodológicos do paradigma construtivista ${ }^{(12)}$.

O Círculo Hermenêutico Dialético foi utilizado como técnica de coleta de dados, método preconizado pela Avaliação de Quarta Geração, Que prevê observação e entrevistas. Através da utilização do círculo hermenêutico-dialético, as primeiras entrevistas foram menos estruturadas, permitindo Que o respondente falasse livremente sobre o serviço. Mas, à medida Que as entrevistas foram realizadas, a análise permitiu Que fossem identificadas Questões expressas nas entrevistas seguintes, de modo Que, cada vez mais, as entrevistas se estruturassem sem deixar de permitir Que todos os entrevistados pudessem lançar novas Questões se assim o desejassem. Foram entrevistados doze familiares.

A escolha dos familiares entrevistados foi intencional, pois se tinha interesse em trabalhar com famílias Que apresentassem algum nível de inserção no serviço. Teve-se a preocupação entrevistar familiares Que não tivessem boa inserção no serviço, uma forma de incluir no círculo hermenêutico as mais diversas opiniões.

As entrevistas foram agendadas previamente de acordo com o dia, hora e local marcados pelos próprios entrevistados. Todas foram gravadas e em seguida ouvidas, a fim de introduzir no círculo hermenêutico Questões/assuntos na próxima entrevista. As primeiras entrevistas foram mais curtas e as últimas mais longas, pois estas contemplavam Quase a maioria dos assuntos introduzidos no círculo.

A etapa de análise dos dados procedeu-se através de três etapas. A primeira etapa é a ordenação dos dados, sendo esta ordenação uma tentativa de estabelecer uma primeira classificação para a análise. Iniciou-se, então, a segunda etapa, a de classificação dos dados feita através de uma leitura exaustiva e repetida dos textos das entrevistas e observações. Após, foi realizada uma leitura transversal para produzir a formação de núcleos temáticos, a fim de conseguir análises e interpretações mais detalhadas. Na análise dos dados foi feita a partir das unidades de registro em confronto com a bibliografia disponível sobre o assunto, e com base na experiência e interpretação pessoal prévia ${ }^{(13)}$.

Participaram do estudo os sujeitos Que concordaram em participar e assinar o Termo de Consentimento Livre e Informado, autorizando a gravação das entrevistas e oue estavam de acordo com o critério de inclusão. Os critérios de seleção dos participantes foram os familiares Que apresentaram algum nível de inserção no serviço e Que fossem maiores de 18 anos. Foi utilizado o Termo de Consentimento Livre e Informado em duas vias, de acordo com a Resolução 196/96 do Conselho Nacional de Saúde, Que aprovam diretrizes e normas regulamentadoras de pesquisa envolvendo seres humanos $^{(14)}$.

Este estudo foi aprovado pelo Comitê de Ética em Pesquisa da cidade de Pelotas, RS, parecer número 074/2005.

\section{RESULTADOS}

O CAPS, objeto desse estudo, foi inaugurado em 16 de agosto de 1988, para atender pessoas com transtornos mentais, sendo subsidiada por recursos da Prefeitura Municipal em pareceria com os governos estadual e federal, conforme o atual Projeto Terapêutico do $\mathrm{CAPS}^{(15)}$.Esse serviço foi um dos pioneiros na Região Sul do Brasil, o Que mostra a relevância deste estudo por centrar-se nesse espaço.

Este serviço não apresenta um aspecto de hospital psiQuiátrico, 
por exemplo, até porque um dos seus objetivos principais é justamente substituir essa instituição total. O ambiente do CAPS é favorável ao acolhimento, à integralidade das ações em saúde, preservando e fortalecendo os laços sociais dos usuários em seu território. Não basta apenas um ambiente interno preservado e aconchegante, mas é necessário um local externamente atraente.

Nesse cenário, os usuários podem ser enQuadrados em três tipos de modalidades de atendimento: intensivo, semi-intensivo e não intensivo. A modalidade é definida conforme a necessidade do usuário, e as sugestões da equipe são discutidas em reunião. Segundo dados da Coordenação do CAPS $^{(15)}$, havia vinte e um usuários intensivos, trinta e seis semi-intensivos e trinta e dois nãointensivos, totalizando cento e nove pacientes. As modalidades de atendimento não são enQuadres estáticos, pois, durante as reuniões de equipe, os casos são discutidos e se houver necessidade a freqüência dos usuários pode mudar.

O momento do acolhimento é de extrema importância no tratamento do usuário e na participação do familiar no CAPS, pois é o primeiro contato Que estes têm com o serviço. A preparação da equipe é fundamental para a vinculação do usuário e da sua família no serviço, sendo preciso agir com receptividade e empatia.

$\mathrm{O}$ acolhimento no CAPS ocorre diariamente, em um sistema de rodízio entre os profissionais responsáveis, destacando-se: assistente social, psicóloga e médico. O Projeto Terapêutico do serviço aborda somente o acolhimento direcionado aos usuários, em nenhum momento fala sobre o acolhimento Que deve ser destinado aos familiares desse usuário.

Muitas vezes chega ao serviço não o usuário, mas o familiar. Então o familiar também é atendido. Também deve ser acolhido. (Família 4)

O acolhimento está ligado ao processo de trabalho em saúde de forma a atender a todos os Que procuram os serviços de assistência à saúde, assumindo uma postura acolhedora, de escuta e de dar respostas adequadas à necessidade de cada sujeito ${ }^{(16)}$.

Entende-se que no momento do acolhimento o profissional responsável deve ter uma ação resolutiva. Ter uma ação resolutiva não se limita a uma conduta clínica, consiste, também, em oferecer possibilidades de usar tudo o Que se dispõe para eliminar o sofrimento e as causas reais do problema do paciente e/ou do seu familiar. Eles devem sair do serviço certo de Que foi feito tudo para resolver seus problemas e Que a equipe de saúde está engajada nas suas causas ${ }^{(17)}$.

É de extrema importância, nesse contexto, saber de Que modo os familiares caracterizam a atenção dispensada pelos profissionais do serviço.

O atendimento eu acho muito bom, muito especial. (Família I)

O atendimento é muito bom, agente chega lá, e é bem recebida, bem tratada, são bem simpáticas. Pra mim é ótimo o atendimento lá. (Família 3)

Os familiares caracterizam a atenção oferecida pelo serviço como sendo "especial, ótimo, muito bom", pois se sentem "bem tratados, bem recebidos" no serviço. Um familiar ressalta Que os profissionais o "abraçam", o Que denota um carinho recebido pelo entrevistado. A relação de ajuda ocorre Quando há o encontro de duas pessoas, o ajudador acolhe o ajudado, deste modo, acolher significa receber o ajudado, transmitindo-lhe receptividade e interesse de modo Que se sinta valorizado ${ }^{(18)}$. A partir das falas dos entrevistados nota-se Que o CAPS oferece uma atenção diferenciada, pautada na humanização, no contato humano, na valorização de aspectos subjetivos.

Observa-se Que a atenção em saúde está atrelada ao cuidado humanizado integral, ao acolhimento e à criação de vínculo. Se todas essas premissas forem contempladas pelos trabalhadores em saúde, melhorará a Qualidade dos serviços de saúde, satisfazendo os usuários e familiares. A atenção à saúde e o sistema de saúde objetivam conferir aos indivíduos, família e comunidade cuidado humanizado nas ações e serviços de saúde, ampliando máxima Qualidade de assistência, acolhimento das pessoas e a resolutividade de cada caso ${ }^{(10)}$.

Segundo o Projeto Terapêutico do CAPS estudado, o Grupo com Familiares de usuários é coordenado por uma assistente social com a participação de um acompanhante terapêutico, tendo um caráter informativo, terapêutico e de auxílio nas Questões de manejo e cuidado com a medicação dos usuários Quando em sua resistência e de orientação para administrar as dificuldades inerentes à condição dos usuários ${ }^{(15)}$.

Eles nos fazem enxergar, Que o nosso problema não é o pior de todos, e nos ensinam como lida com eles, a ter mais paciência também com limite, não assim liberando tudo, não aceitando tudo, como te calma. Aí fica um ambiente mais saudável, um ambiente mais amistoso dentro de casa, todo mundo acaba tendo mais paciência, tanto pra lidar pro outro, Quanto até por si mesmo, pensa um pouQuinho mais antes de agir, não agi tão impulsivamente. (Família 6)

O grupo de apoio para familiares consiste num espaço terapêutico Que possibilita ao familiar um entendimento sobre o diagnóstico, expressões da doença e a compreensão do tratamento com enfoque psicossocial. Auxilia, também, a melhor o desempenho dos pacientes em casa e em suas relações sociais e permite um momento para expressarem suas emoções diante das desadaptações dos pacientes, fornecendo trocas para ajustamentos ante essas emoções ${ }^{(19)}$.

Durante a coleta de dados no serviço não foi presenciado um atendimento individual aos familiares, o Que foi evidenciado é Que no grupo semanal destinado às famílias, apenas um familiar compareceu, e seu interesse estava relacionado ao benefício. A dificuldade revelada pelo serviço em atrair os familiares pode estar relacionada ao pouco preparo dos profissionais ou até a sua falta de conscientização sobre a importância do papel da família no setting terapêutico.

Percebe-se que a equipe do CAPS estudado encontra dificuldades em fazer com Que a família participe do cotidiano do serviço. Para Que essa unidade de cuidado participe e vivencie o dia-a-dia do CAPS é preciso Que a família sinta-se ator importante no processo de reabilitação psicossocial do seu paciente.

Outro recurso Que o CAPS disponibiliza para estar mais próximo do ambiente familiar do usuário são as visitas domiciliares, realizadas, pelo menos, uma vez por mês a todos os usuários ou 
Quando a equipe julgar necessário, e também por solicitação do familiar ou do próprio usuário.

A partir de trechos das entrevistas percebe-se Que as visitas domiciliares são de extrema importância para o acompanhamento do usuário e também de sua família.

Dois membros da equipe foram fazer uma visita na minha casa,com o objetivo de saber como o meu familiar fica em casa, como a gente ajuda ele. (Família7)

A visita domiciliar é um dos instrumentos mais indicados para a prestação de assistência à saúde, do indivíduo, família e comunidade. Apesar de antiga, a visita domiciliar traz resultados inovadores, porque possibilita conhecer a realidade do cliente e sua família in loco, além de fortalecer os vínculos cliente, terapêutica e profissional $^{(20)}$. Assim, percebe-se Que a visita domiciliar não foi utilizada apenas para oferecer suporte ao usuário, mas também para dar apoio ao familiar. A visita domiciliar também consiste em um espaço para trabalhar com a psicoeducação e para aproximar usuário-família-serviço.

As formas usadas pela equipe do CAPS para se comunicar com os familiares dos usuários, e estes, com o serviço, são descritas abaixo:

Ah, marcam as reuniões tudo direitinho, mandam bilhete, com antecedência [...] ou eu telefono. É, eles Que mandam pra mim os bilhetinhos. (Família 12).

Através do telefone, ligando, ou eu vou até ali e converso com eles. É Que aqui é tão pertinho, é mais fácil a gente ir ali do que às vezes telefonar. (Família7)

Fica evidente Que a principal forma Que o serviço utiliza para se comunicar com as famílias é através de bilhetes entregues pelo usuário. lá, as famílias, por sua vez, preferem utilizar o telefone para entrar em contato com o serviço ou ir direto à sede do CAPS. O fato de cobrar a presença da família no serviço faz parte de um processo de responsabilização da família com o seu paciente, preconizado pela Reforma PsiQuiátrica.

Outra atividade que inclui os familiares consiste na Associação dos Familiares e Usuários do Serviço (AFAU), fundada em 1992. A AFAU surgiu justamente para suprir a necessidade do serviço em trazer o familiar para dentro do CAPS, tendo em vista certa relutância dos familiares em participarem do dia-a-dia do serviço.

As associações de familiares são uma identidade coletiva norteada, principalmente, por uma ação política dirigida à conQuista de maior visibilidade social, tanto por parte dos usuários Quanto dos familiares ${ }^{(21)}$.

Uma nova abordagem aos familiares começou a ser pensada após um evento estadual de Saúde Mental. Após esse encontro, vários profissionais mencionaram ter participado de uma palestra sobre a importância dos serviços de saúde mental proporcionarem espaços em Que os familiares possam falar sobre os seus sentimentos, suas angústias e dificuldades em lidar com os seus pacientes. A idéia de fazer um grupo especificamente para os familiares foi aceita e bem vista tanto pelos usuários, familiares Quanto pela equipe.

A secretária fala Que depois de um evento que participaram, começaram a pensar numa nova abordagem aos familiares, não mais, falando apenas do cuidado com seu ente, mas sim, voltada para o sofrimento e cuidado dos próprios [...] (Observação2).

É um desafio para os serviços de saúde "cuidar de Quem cuida”, ou seja, ir ao encontro da família, conhecendo como cuida, identificando suas dificuldades e suas forças para melhor atuação profissional, através do conhecimento das necessidades familiares ${ }^{(7)}$.

A introdução ativa do familiar no setting terapêutico aparece como uma prática e uma filosofia do Serviço. Desta forma, a atenção dispensada aos familiares pelo CAPS encontra-se presente no diaa-dia do serviço, ainda Que seja incipiente. Considera-se Que a atenção aos familiares a ser desenvolvida no CAPS necessita dar conta da complexidade das relações entre seres humanos a partir de novas abordagens.

Nessa perspectiva, acredita-se necessária a conscientização e a mobilização por parte dos profissionais da equipe sobre a importância da participação da família no serviço, a fim de desencadear uma atenção ainda mais humana, integral e individualizada aos familiares.

\section{CONSIDERAÇÕES FINAIS}

Percebe-se que o CAPS estudado encontra dificuldades em fazer com Que a família participe do cotidiano do serviço. Para isso é preciso que a mesma se sinta um ator importante no processo de reabilitação psicossocial do seu paciente. Este é um processo de construção cotidiana, na Qual são superadas visões e posturas, conduzindo a movimentos Que promovem um entrelaçamento Que se refletirá em melhor Qualidade da atenção dispensada ao usuário e sua família.

Constatou-se que a família é um parceiro no cuidado, facilitando o processo de reabilitação psicossocial do sujeito com transtorno mental. É importante salientar Que não é só a família Que possui um papel neste contexto, mas também o serviço Que deve fazer um movimento para atrair e atender as necessidades desses agentes facilitadores do cuidado.

Ressaltamos a relevância deste estudo, pois contribuiu para as concepções das famílias acerca da atenção oferecida aos familiares pelo CAPS, articulando teorias e práticas, propondo uma avaliação Qualitativa, mostrando-lhes sua importância no direcionamento de melhorias na Qualidade dos serviços prestados.

As práticas dos serviços de Saúde Mental devem ser pautadas nos pressupostos da Reforma Psiquiátrica, como forma de Qualificar $a$ atenção oferecida às famílias. A forma como vem sendo consolidada a atenção às famílias nos serviços representa um desafio para os profissionais de saúde, pois implica em uma reestruturação na formação desses e um processo de reflexão de todos os atores sociais envolvidos neste processo de mudança. 


\section{REFERÊNCIAS}

1. Novaes HMD. Avaliação de programas, serviços e tecnologias em saúde. Rev Saúde Pública 2000; 34(5):547-9.

2. Almeida PF, Escorel S. Da avaliação em saúde à avaliação em Saúde Mental: gênese, aproximações teóricas e Questões atuais. Saúde em Debate 2001; 25(58): 35-47.

3. Hartz ZMA, Pouvourville G. Avaliação da eficiência em saúde: a eficiência em Questão. Ciên Saúde Coletiva 1998; 3(1): 6882.

4. Onocko-Campo RT, Furtado IP. Entre a saúde e a saúde mental: um instrumental metodológico para avaliação da rede de Centros de Atenção Psicossocial (CAPS) do Sistema Único de Saúde. Cad Saúde Pública 2006; 22(5): 1053-62.

5. Ministério da Saúde (BR). Secretaria Executiva. Núcleo Técnico da Política Nacional de Humanização. HumanizaSUS: Política Nacional de Humanização: a humanização como eixo norteador das práticas de atenção e gestão em todas as instancias do SUS. Brasília: Ministério da Saúde; 2004.

6. Pejlert A. Being a parent of an adult son or daughter with severe mental illness receiving professional care: parents narratives. Health Social Care Comm 200 I; 9(4): 194-204.

7. Elsen I, Nitschke RG. Saúde da família na pós-graduação: um compromisso ético interdisciplinar na pós modernidade. Rev Bras Enferm 2000; 53(esp): 35-48.

8. Pessoti I. O século dos manicômios. São Paulo: Editora 34; 1996.

9. Ministério da Saúde (BR). Conselho Nacional de Saúde. Cuidar sim, excluir não: efetivando a Reforma PsiQuiátrica com acesso, Qualidade, humanização e controle social: relatório final. In: Conferência Nacional de Saúde Mental, 200I; Brasília (DF), Brasil. Brasília: Ministério da Saúde; 2002.

10. Prefeitura Municipal de Goiânia (GO). Secretaria Municipal de Saúde. Controle social no SUS: construindo para conquistar saúde. In: Conferência Municipal de Saúde, 200I, Goiânia (GO), Brasil. Goiânia: Secretaria Municipal de Saúde; 2001 .
11. Ministério da Saúde (BR). Portaria n. ${ }^{\circ}$ 336/GM, de 19 de fevereiro de 2002. Dispõe sobre a proteção e os direitos das pessoas portadoras de transtornos mentais e redireciona o modelo assistencial em saúde mental. Brasília: Ministério da Saúde; 2002

12. Guba E, Lincoln Y. Fourth generation evaluation. Newbury Park: Sage; 1989

13. Minayo MCS. O desafio do conhecimento: pesquisa Qualitativa em saúde. São Paulo: Hucitec/Abrasco; 1993.

14. Ministério da Saúde (BR). Conselho Nacional de Saúde. Comitê Nacional de Ética em Pesquisa em Seres Humanos. Resolução $n^{\circ} 196$, de 10 de outubro de 1996. Dispõe sobre as diretrizes e normas regulamentadoras de pesquisa envolvendo seres humanos. Brasília: Ministério da Saúde; 1996.

15. Prefeitura Municipal de São Lourenço do Sul (RS). Projeto Terapêutico CAPS I - Nossa Casa. São Lourenço do Sul: Prefeitura Municipal de São Lourenço do Sul; 2006.

16. Mello R. A Questão da interdisciplinaridade no dia-a-dia da enfermeira Que atua em centros de atenção diária de Saúde Mental. Rev Bras Enferm 1998; 5 I(1): 19-34.

17. Malta GC. Mudando o processo de trabalho na rede pública: alguns resultados da experiência em Belo Horizonte. Saúde em Debate 2000; 4 (17): 47-58.

18. Miranda CF, Miranda ML. Construindo a relação de ajuda. Belo Horizonte: Crescer; 2002.

19. Duarte MLC. Diferentes abordagens à família em saúde mental presentes na produção científica da área. Rev Mineira Enferm 2007; II(I): 66-72.

20. Souza RC, Lopes SCF, Barbosa MA. A contribuição do enfermeiro no contexto de promoção à saúde através da visita domiciliar. Revista UFG 2004; 6(esp).

21 . Souza WS. Associações civis em saúde mental no Rio de Janeiro: democratizando os espaços sociais. Cad Saúde Pública 200 I; 17(4): 933-9. 